\title{
The Biosensing of Microparticles: Benefits and Perspectives
}

\author{
Alexander E. Berezin, Professor, MD, PhD. \\ Professor, MD, PhD, Consultant of Cardiology Unit, Internal Medicine Department, State Medical \\ University, 26, Mayakovsky av., Zaporozhye, Ukraine \\ dr_berezin@mail.ru
}

\begin{abstract}
Micro particles (MPs) are defined a heterogeneous population of vesicles (diameter 100-1000 $\mathrm{nm}$ ) that are released by cellular vesiculation and fission of the membrane of cells and play a pivotal role in various diseases including cardiovascular diseases, cancer, sepsis, eclampsia, autoimmune and metabolic states. Currently there is no standardization regarding analytical methods of MP detection. This editorial comment focuses on the current knowledge about MPs and analytical methods of its detection. Conventional methods have crucial limitations regarding complicated assay and suffers from relatively low sensitivity and accuracy because of resolution problems occurring for the majority of commercially available flow cytometers. Alternatively, recently recognized as a method for quantification and sizing of biological nano-particlessurface plasmon resonance - based imaging microscopy (SPRi microscopy) might be significantly useful to resolve the majority problems affected MPs recognition. Probably Raman micro-spectroscopy, micro nuclear magnetic resonance technique, small-angle X-ray scattering, and anomalous small-angle X-ray scattering might compete with SPRi microscopy and flow cytometery. Sort comment is discussed contemporary approaches regarding novel techniques of microparticle determination, measurements and assay.
\end{abstract}

Keywords: Micro particles, Analytical Assay, Flow Cytometry, Western Blot Analysis, Electron Microscopy, Surface Plasmon Resonance-Based Imaging Microscopy, Nuclear Magnetic Resonance Techniques.

Microparticles (MPs) have great potentiality in material science- based applications [1]. Developments of technologies that attenuate recognize, determination, and measurements of MPs obtained from various cells appear to be indispensable tool to clinical medicine [2]. Recent investigations have been shown that MPs as derivates of cellular membrane are discussed powerful paracrine regulators of target cell functions [3-5]. Indeed MPs possess a wide spectrum of biological effects on intercellular communication by transferring different molecules able to modulate other cells affected growth of tissue, reparation, vasculogenesis, inflammation, apoptosis, infection, and malignancy. Growing evidence supports the idea that regarding association between immune pattern of MPs originated from different cells (endothelial cells, mononuclears, dendritic cells, platelets) and nature evolution of various diseases including cardiovascular diseases, cancer, sepsis, eclampsia, autoimmune and metabolic states, etc. [6-9].

MPs are defined a heterogeneous population of vesicles (diameter 100-1000 nm) that are released by cellular vesiculation and fission of the membrane of cells [10]. This mechanism affects genome and may mediate by some triggers [11]. In is well known that MPs appear to be found into circulation in response to many situational changes (physiological conditions, stress) microenvironmental stimulation, coagulation / thrombosis, endotoxinemia, endothelial shear stress, activated cells or those undergoing apoptosis, ischemic injury, hypoxia, and malignancy [12-14].

The current stand of knowledge regarding morphology, transcriptomics, and proteomics of circulating MPs is still not fully $[15,16]$. The difficulty of separating MPs realized from other types of cells limits or efforts to extend actual cognitions in features affected biogenesis, secretion, and subsequent biological role of MPs. Moreover, accurate assessment of MPs remains a serious challenge because of a lack of consensus regarding methodologies to measure MPs and the inability of most techniques to capture the entire size range of these vesicles [17]. Therefore, the MP detection methods used till date are costly and time consuming [18].

The conventional approach for measuring the MPs is based on commonly used flow cytometry and nano-particle tracking analysis (NTA) that recognized as a gold standard, as well as Western blot analysis and electron microscopy. However the definition of MPs using these techniques is still an area of great debate [19]. Unfortunately, all methods have crucial limitations regarding complicated 
assay and suffers from relatively low sensitivity and accuracy because of resolution problems occurring for the majority of commercially available flow cytometers [20, 21]. The next serious barrier created surmountable problems for NTA is sizing of small MPs $(<50-100 \mathrm{~nm})$. The novel approaches that could allow increasing an ability of flow cytometry technique to detect small-size MPs is advanced cytofluorimetric method based on BD Horizon Violet Proliferation dye. However, the utilization of flow cytometers specifically designed for analysis of small-size MPs is probably to provide considerable methodological advantages and should be the preferable options [20]. In addition, problems with concentration limits of NTA measurements restrict the use of this method for clinical samples [21]. Western blot analysis and electron microscopy allow to optionally recognize MPs depending on determination of different markers, represents a useful tool for examining particles. However, Western blot analysis and electron microscopy require subsequent technical efforts and are much expensive.

Alternatively, recently recognized as a method for quantification and sizing of biological nanoparticles- surface plasmon resonance - based imaging microscopy (SPRi microscopy) might be significantly useful to resolve the majority problems affected MPs recognition. SPRi is discussed a highly sensitive label-free biochemical surface sensor measurement technique that has only recently been applied to the field of cell-biology. This method is based on phenomenon known as surface plasmon resonance that associates with a high resolute diffraction generated at a thin metal surface [22]. The high contrast in SPR signal between cell edges and substratum facilitates identification of cell edges and segmentation of cell areas [23]. Importantly that several cells, cellular components (i.e. focal adhesions, nucleus, and cellular secretions), viruses, bacteria, micro- and nano-particles have not just became visible, but they are able to be calculable [22, 23]. As expected, a quantitative interpretation of SPRI imaging might improve resolution of MP determination and allow investigators unprecedented to overcome flow cytometry limitations regarding low detectable small-size MPs [24]. Moreover, simultaneous application of a high-sensitive fluorescent microscopy and SPRi microscopy should enhance the sensitivity and selectivity of a created biosensor platform [25, 26]. This might have a high value for identification of small-size MPs originated from different cells that were recently determined as debris [27]. Probably small-size MPs derived from apoptotic cells play a pivotal role in tissue injure, inversely MPs secreted activated cells, i.e. mononuclears, endothelial cells, dendritic cells, may have a positive effect on tissue repair and homeostasis [28].

A highly sensitive fluorescent (HSF) microscopy also permits to detect individual sub-micro and nano-MPs. As compared with SPRi microscopy, this technique could provide higher detection sensitivity due to a large fluorescence excitation and a high effective quantum yield of fluorescence. Therefore, there are at least four methods that are not commercially available: Raman microspectroscopy, micro nuclear magnetic resonance technique, small-angle $\mathrm{X}$-ray scattering, and anomalous small-angle X-ray scattering [29].

Surface-assisted laser desorption/ionization mass spectrometry (SALDI-MS) is a high throughput analytical technique capable of detecting low molecular weight analytes, including MPs [30]. The main advantage of this novel approach is possibility for the analysis of MPs isolated from multiple biological fluids without sample preparation protocols. In this setting the effect of MP size, pore diameter, pore depth and "functionalization" on analytical performance might be studied. In fact, the method detection limits are 10-30 nm that opens novel perspectives in investigations of small-size MPs. However the role of SALDI-MS in biosensing of MPs is not fully investigated and requires scrutiny.

There are commercial platforms that offer massively parallel, label-free biosensing of MP based on combining all-electrical detection with low-cost integrated circuits [31]. Despite these successes, bioelectronics has so far failed to deliver a broadly applicable MP biosensing platform in routine practice. All these methods are currently being explored to assay MPs, while an incorporation of these techniques into analytical care is probably addressed in the future.

In conclusion, a standardization of the methods of nano- and micro- particles determination is extremely required. Commonly used procedures, such as flow cytometry with NTA, Western blot analysis, SALDI-MS, electron microscopy, and other methods might not have universal utility for MP determination; especially for small-size MPs. Novel techniques regarding identification of MPs based on real-time and label-free optical biosensors and principles of SPR phenomena appear to be much attractive and could sufficiently overcome limitation of option methods of MP determination. 


\section{REFERENCES}

Sarkar S, Dasgupta AK. Microparticle of drug and nanoparticle: a biosynthetic route. Pharmacol Res Perspect. 2015; 3(5): e00188.

Gu Z, Jing C, Ying YL, He P, Long YT. In situ high throughput scattering light analysis of single plasmonic nanoparticles in living cells. Theranostics. 2015; 5(2): 188-95.

Gong J, Jaiswal R, Dalla P, Luk F, Bebawy M. Microparticles in cancer: A review of recent developments and the potential for clinical application. Semin Cell Dev Biol. 2015; 40: 35-40.

Das S, Halushka MK. Extracellular vesicle microRNA transfer in cardiovascular disease. Cardiovasc Pathol. 2015; 24(4): 199-206.

Berezin A, Zulli A, Kerrigan S, Petrovic D, Kruzliak P. Predictive role of circulating endothelialderived microparticles in cardiovascular diseases. Clin Biochem. 2015; 48(9): 562-568.

Jadli A, Sharma N, Damania K, Satoskar P, Bansal V, Ghosh K, et al. Promising prognostic markers of Preeclampsia: New avenues in waiting. Thromb Res. 2015. pii: S0049-3848(15)00238-8. doi: 10.1016/j.thromres.2015.05.011. [Epub ahead of print]

Greening DW, Gopal SK, Mathias RA, Liu L, Sheng J, Zhu HJ, et al. Emerging roles of exosomes during epithelial-mesenchymal transition and cancer progression. Semin Cell Dev Biol. 2015; 40: 60-71.

Martinez MC, Andriantsitohaina R. Microparticles in angiogenesis: therapeutic potential. Circ Res. 2011; 109: 110-119

Souza AC, Yuen PS, Star RA. Microparticles: markers and mediators of sepsis-induced microvascular dysfunction, immunosuppression, and AKI. Kidney Int. 2015; 87(6): 1100-1108.

Colombo M, Raposo G, Théry C. Biogenesis, secretion, and intercellular interactions of exosomes and other extracellular vesicles. Annu Rev Cell Dev Biol. 2014; 30: 255-89.

Piccin A, Murphy WG, Smith OP. Circulating microparticles: pathophysiology and clinical implications. Blood Rev. 2007; 21: 157-71.

Lynch SF, Ludlam CA. Plasma microparti-cles and vascular disorders. Br J Haematol. 2007; 137: 3648.

Sabatier F, Camoin-Jau L, Anfosso F, Sampol J, Dignat-George F. Circulating endothelial cells, microparticles and progenitors: key players towards the definition of vascular competence. J Cell Mol Med. 2009; 13(3):454-71.

Burnier L1, Fontana P, Kwak BR, Angelillo-Scherrer A. Cell-derived microparticles in haemostasis and vascular medicine. Thromb Haemost. 2009; 101(3): 439-51.

Inal JM, Kosgodage U, Azam S, Stratton D, Antwi-Baffour S, Lange S. Blood/plasma secretome and microvesicles. Biochim Biophys Acta. 2013; 1834(11): 2317-25.

Choi DS, Kim DK, Kim YK, Gho YS. Proteomics, transcriptomics and lipidomics of exosomes and ectosomes. Proteomics. 2013; 13(10-11): 1554-71.

Dinkla S, Brock R, Joosten I, Bosman GJ. Gateway to understanding microparticles: standardized isolation and identification of plasma membrane-derived vesicles. Nanomedicine (Lond). 2013; 8(10): 1657-68.

Patil R, Ghosh K, Shetty S. A simple clot based assay for detection of procoagulant cell-derived microparticles. Clin Chem Lab Med. 2015 pii: /j/cclm.ahead-of-print/cclm-2015-0508/cclm2015-0508.xml. doi: 10.1515/cclm-2015-0508. [Epub ahead of print]

Niccolai E, Squatrito D, Emmi G, Silvestri E, Emmi L, Ciucciarelli L, et al. A new cytofluorimetric approach to evaluate the circulating microparticles in subjects with antiphospholipid antibodies. Thromb Res. 2015. doi: 10.1016/j.thromres.2015.10.018. [Epub ahead of print]

Shantsila E, Montoro-García S, Gallego P, Lip GY. Circulating microparticles: challenges and perspectives of flow cytometric assessment. Thromb Haemost. 2014; 111(6):1009-14.

Orozco AF, Lewis DE. Flow cytometric analysis of circulating microparticles in plasma. Cytometry A. 2010; 77(6):502-14.

Peterson AW, Halter M, Tona A, Plant AL. High resolution surface plasmon resonance imaging for single cells. BMC Cell Biol. 2014; 15: 35.

Peterson AW, Halter M, Tona A, Bhadriraju K, Plant AL. Using surface plasmon resonance imaging to probe dynamic interactions between cells and extracellular matrix. Cytometry A. 2010; 77(9):895-903. 
Robelek R, Wegener J. Label-free and time-resolved measurements of cell volume changes by surface plasmon resonance (SPR) spectroscopy. Biosens Bioelectron. 2010; 25(5): 1221-4.

Shpacovitch V. Application of Surface Plasmon Resonance (SPR) for the Detection of Single Viruses and Single Biological Nano-objects. J Bacteriology and Parasitology. 2012; 3: e110.

Zybin A, Kuritsyn YA, Gurevich EL, Temchura VV, Überla K, et al. Real-time detection of single immobilized nanoparticles by surface plasmon resonance imaging. Plasmonics. 2010; 5: 31-35.

Berezin AE, Kremzer A.A. SamuraT.A., Berezina T.A, Kruzliak P. Impaired immune phenotype of circulating endothelial-derived microparticles in patients with metabolic syndrome and diabetes mellitus. J Endocrinol Invest. 2015; 38(8): 865-74.

Berezin AE. Impaired Phenotype of Circulating Endothelial-Derived Microparticles: Novel Marker of Cardiovascular Risk. J Cardiology and Therapy. 2015; 2(4): 273-278

van der Pol E, Coumans F, Varga Z, Krumrey M, Nieuwland R. Innovation in detection of microparticles and exosomes. J Thromb Haemost. 2013; 11 Suppl 1: 36-45.

Guinan TM, Kirkbride P, Della Vedova CB, Kershaw SG, Kobus H, Voelcker NH. Direct detection of illicit drugs from biological fluids by desorption/ionization mass spectrometry with nanoporous silicon microparticles. Analyst. 2015. [Epub ahead of print]

Laborde C, Pittino F, Verhoeven HA, Lemay SG, Selmi L, Jongsma MA, et al. Real-time imaging of microparticles and living cells with CMOS nanocapacitor arrays. Nat Nanotechnol. 2015; 10(9): $791-5$. 